\title{
Sixth Sense Technology
}

\author{
http://dx.doi.org/10.3991/ijes.v2i4.3883 \\ L.Haritha Sridevi, \\ Computer Science Engineering, Saveetha School of Engineering, India
}

\begin{abstract}
Sixth sense is a gestural interface gadget representing a neck-worn pendant that holds both an information projector and Polaroid. Head-worn alternatives were additionally constructed at MIT Media Lab in 1997 that consolidated Polaroids and light frameworks for intelligent photographic craftsmanship, and likewise included signal distinguishment (e.g. finger-following utilizing hued tape which are worn on the fingers)
\end{abstract}

Sixth-sense is a name for additional data supplied by a wearable workstation, for example, the gadget called "WUW" (Wear your World) by Pranav Mistry expanding on the idea of the Telepointer, a neckworn projector and Polaroid mix initially proposed and decreased to practice by MIT Media Lab scholar Steve Mann.

Index Terms - sixth sense, gestural interface, wearable

\section{DEFINITION}

Sixth Sense Technology organizes advanced data into the physical world and its disputes, making the whole world your workstation. It has the ability to transform any surface into a touch-screen for processing and controlled by basic hand motions. It is not an engineering which changes human propensities yet bringing about workstations and different machines to adjust to the human needs. It additionally reverses multi- client and multi touch results. Sixth Sense gadget is a small projector coupled with a Polaroid and a PDA which goes about as the machine and your association with the Cloud, all the data put away on the web. The current model sets back the finances around $\$ 350$. The Sixth Sense model is utilized to realize a few necessities that have demonstrated the convenience, reasonability and adaptability of the framework.

\section{CAUSE}

Sixth Sense innovation i.e.Polaroid combined with light source was created in 1997 (head-worn) and 1998 (neckworn), however the Sixth Sense name for this work was at first proven and spread in 2001. Mann hinted this wearable believing improvement as bearing a "Produced Synesthesia of the Sixth Sense", i.e. the possibility that wearable handling and drove information can go about as an additional (i.e. sixth sense) ten years after, Pattie Maes (furthermore with MIT Media Lab) used the analysis "Sixth Sense" in this same setting, in her TED conversation.

\section{DEVELOPMENT AND WORKING}

The Sixth sense innovation embraces a pocket projector, a mirror and a Polaroid held in a head-mounted, handheld or pendant-like, wearable gadget. Both the projector and the Polaroid are linked with a versatile figuring

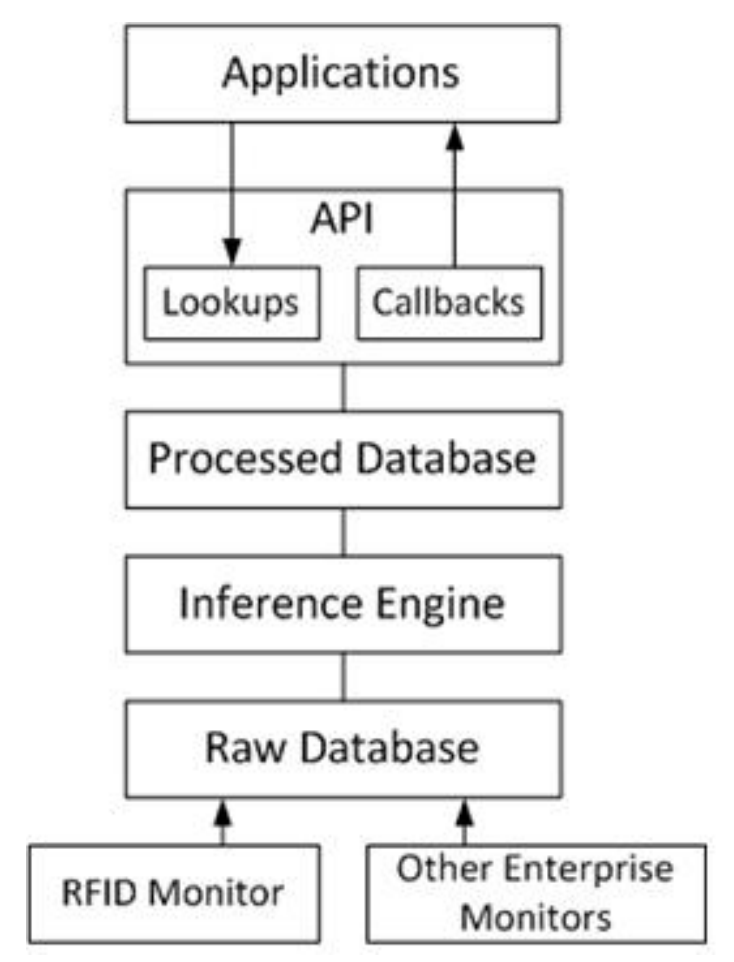

Figure 1.

gadget in the client's pocket. The projector endeavors visual data allowing surfaces, dividers and physical questions around us to be utilized as interfaces; while the Polaroid distinguishes and tracks clients' hand signals and physical items utilizing workstation vision based actions. The product system forms the feature stream information caught by the Polaroid and tracks the areas of the shaded markers (visual following fiducials) at the tips of the client's fingers. The developments and plans are translated into signals that demonstration as teamwork directions for the expected provision interfaces.

\section{SAMPLE APPLiCATIONS}

Throughout the TED talk given by Professor Pattie Maes, she demonstrated a feature exhibiting various demands of the Sixth-sense framework:

1. Four hued cursors are controlled by four fingers wearing diverse shaded markers continuously. The projector shows feature sentiment to the client on a vertical divider.

2. The projector shows a guide on the divider, and the client can control the guide utilizing zoom and skillet signals.

3. The client can make an edge signal to train the Polaroid to take a picture. It is implied that the photo- 
graph will be consequently edited to uproot the client's hands.

4. The framework could extend numerous photographs on a divider, and the client could sort, re-estimate and arrange them with signals. This provision was called Reality Window Manager (RWM) in Mann's head-worn usage of Sixth Sense.

5. A number cushion is projected onto the client's palm, and the client can dial a telephone number by touching his palm with a finger. It was indicated that the framework can stick point the area of the palm. It was additionally indicated the Polaroid and projector can alter themselves for surfaces that are not level.

6. The client can get an item in market (e.g. a bundle of paper towels), and the framework could show related data (e.g. the measure of dye utilized) once more on the item itself.

7. The framework can distinguish any book grabbed by the client and presentation Amazon rating on the book spread.

8. As the client opens a book, the framework can show extra data, for example, spectator's remarks.

9. The framework can distinguish distinct pages of a book and presentation explanation by the client's companion. This demo additionally indicated the framework's capability to handle tilted surface.

10. The framework can distinguish daily paper articles and venture the latest feature on the news occasion on a clear locale of the daily paper.

11. The agenda can differentiate individuals by their appearances and undertaking an expression billow of related data recovered from the web on the individual's body.

12. The framework can distinguish a ticket and presentation related data, for example, flight defer and door change.

13. The customer can draw a ring on his wrist, and the framework will extend a clock on it. Note this demo implied at the capacity to exactly discover the area of the wrist.

Notwithstanding wearing the gadget throughout the presentation, Professor Maes did not give a live demonstration of the innovation. Throughout the discussion, she had underlined over and over that the Sixth-sense engineering was a work in advancement, then again it was never.

The Working Of The Sixth Sense Device

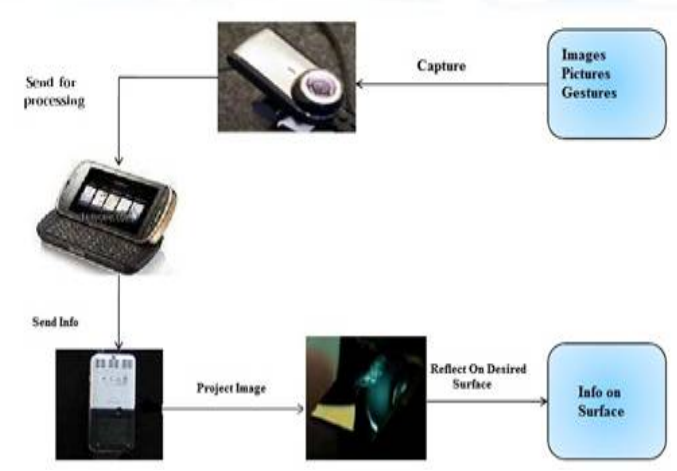

Figure 2.

\section{AdVAntages}

Sixth Sense is an easy to use interface which classifies advanced data into the physical world and its questions, making the whole world your machine.

Sixth Sense does not change human predilections yet causes machine and different machines to adjust to human needs.

It uses hand motions to interface with computerized data, helps multi Touch and multi-client cooperation.

Information gets straightforwardly from machine in real time. It is an open source and savvy and we can brain outline thought anyplace.

It is signaling controlled wearable figuring gadget that nurtures our applicable data and transforms any surface into an intuitive presentation.

It is compacted and simple to convey as we can wear it in our neck.

The gadget could be utilized by anybody without even an essential information of a console or mouse. There is no compelling reason to convey a Polaroid any longer.

In the event that we are striving for an occasion, then from now on wards it will be not difficult to catch photographs by utilizing insignificant fingers

\section{CONCLUSION}

Sixth Sense is a wearable gadget that empowers you to have the entire universe of advanced data readily available truly!

This sets data free and the entire world is your screen or PC.

It has been ordered under the class wearable registering.

The accurate force of Sixth Sense lies on its possibility to unite this present reality with the Internet, and overlaying the data on the world itself.

The key here is that Sixth Sense distinguishes the items around you, showing data consequently furthermore letting you get to it in any capacity you need, in the easiest way possible.

Unmistakably, this has the latent of turning into a definitive "transparent" client interface for getting to data about everything around us. On the off chance that they can dispose of the shaded finger tops and it ever goes past the introductory advancement stage, that is. Anyway as it is currently, it may change the way we communicate with this present authenticity and honestly give everybody complete familiarity with nature

\section{REFERENCES}

[1] http://www.seminarsonly.com//Sixth-Sense-Technology.php

[2] http://www.engineersgarage.com/articles/sixth-sense-technology

[3] Sixth Technology by Mohammed Moteen Tahir

[4] Sixth Sense by Stuart Wilde

[5] Ituitive Mind by Eugene Sadler-Smith

\section{AUTHOR}

L. Haritha Sridevi is with Computer Science Engineering, Saveetha School of Engineering, India.

Submitted 16 May 2014. Published as resubmitted by the author 25 October 2014. 